\title{
NONEXISTENCE OF NONCONSTANT SOLUTIONS OF SOME DEGENERATE BELLMAN EQUATIONS AND APPLICATIONS TO STOCHASTIC CONTROL *
}

\author{
Martino Bardi $^{1}$, Annalisa Cesaroni ${ }^{1,2}$ And Luca Rossi ${ }^{1}$
}

\begin{abstract}
For a class of Bellman equations in bounded domains we prove that sub- and supersolutions whose growth at the boundary is suitably controlled must be constant. The ellipticity of the operator is assumed to degenerate at the boundary and a condition involving also the drift is further imposed. We apply this result to stochastic control problems, in particular to an exit problem and to the small discount limit related with ergodic control with state constraints. In this context, our condition on the behavior of the operator near the boundary ensures some invariance property of the domain for the associated controlled diffusion process.
\end{abstract}

Mathematics Subject Classification. 35J60, 35J70, 93E20, 35B53.

Received December 30, 2014. Revised May 6, 2015.

\section{INTRODUCTION}

We consider the fully nonlinear elliptic operator

$$
F[u]:=\sup _{\alpha \in A}\left(-b(x, \alpha) \cdot D u(x)-\operatorname{tr}\left(a(x, \alpha) D^{2} u(x)\right)\right),
$$

which is usually called Hamilton-Jacobi-Bellman (briefly, HJB). Here "tr" denotes the trace of a matrix. Our first main result is in some sense a counterpart for a bounded domain $\Omega \subseteq \mathbb{R}^{n}$ of results of Liouville type in the whole space. We assume that $F$ degenerates at the boundary of $\Omega$, at least in the normal direction to $\partial \Omega$, for some $\bar{\alpha} \in A$, and that the quantity $b(x, \bar{\alpha}) \cdot \operatorname{Dd}(x)+\operatorname{tr}\left(a(x, \bar{\alpha}) D^{2} d(x)\right)$ is positive near $\partial \Omega$, where $d(x)$ is the distance of $x$ from $\partial \Omega$. This is related with the viability, or weak invariance, of $\Omega$ for the controlled diffusion process associated with the operator $F$. We show that any viscosity subsolution $u$ of $F[u]=0$ in $\Omega$ such that

$$
u(x)=o(-\log (d(x))) \quad \text { as } x \rightarrow \partial \Omega,
$$

Keywords and phrases. Hamilton-Jacobi-Bellman equations, degenerate elliptic PDEs, stochastic control, exit-time problems, ergodic control with state constraints, viscosity solutions.

* M.B. and A.C. were partially supported by the European Project Marie Curie ITN "SADCO - Sensitivity Analysis for Deterministic Controller Design". A.C. and L.R. were partially supported by the GNAMPA Project 2014 "Propagation phenomena on lines and networks".

1 Department of Mathematics, University of Padova, Via Trieste 63, 35121 Padova, Italy. bardi@math.unipd.it annalisa.cesaroni@unipd.it; lucar@math.unipd.it

2 Department of Statistical Sciences, University of Padova, Italy. 
must be constant. A similar result holds for supersolutions under a stronger condition on the coefficients near the boundary related to the invariance of the associated diffusion process for all controls.

Results of nonexistence of nonconstant solutions in unbounded domains for elliptic equations are usually called Liouville-type theorems. For fully nonlinear equations they have been studied in the last ten years by several authors, see, e.g., $[18,20]$, and the references therein. They are very different from our result for various reasons. In particular, in our case the drift term compensates the degenerate ellipticity of $F$ at the boundary, whereas in the unbounded case with uniformly elliptic $F$ the drift term represents a difficulty.

The main application of our Liouville-type results concerns the well-posedness of the so-called ergodic Hamilton-Jacobi-Bellman equation

$$
\sup _{\alpha \in A}\left(-b(x, \alpha) \cdot D \chi(x)-\operatorname{tr}\left(a(x, \alpha) D^{2} \chi(x)\right)-l(x, \alpha)\right)=c, \quad x \in \Omega .
$$

The unknowns here are $(c, \chi) \in \mathbb{R} \times C(\Omega)$. Under mild continuity and boundedness assumptions on the data and the non-degeneracy condition in the interior of $\Omega$

$$
a(x, \alpha)>0 \text { for every } x \in \Omega \text { and } \alpha \in A,
$$

we prove that this problem has a solution, the additive eigenvalue $c$ is unique, and the viscosity solution $\chi$ is unique up to the addition of constants among functions $u$ satisfying the boundary condition (1.2).

There are several motivations for the above mentioned results, especially from stochastic optimal control. Here we give two applications, in Sections 5 and 6.2, respectively. The first concerns an exit time problem for the controlled diffusion

$$
\left\{\begin{array}{l}
\mathrm{d} X_{t}^{\alpha .}=b\left(X_{t}^{\alpha .}, \alpha_{t}\right) \mathrm{d} t+\sqrt{2} \sigma\left(X_{t}^{\alpha .}, \alpha_{t}\right) \mathrm{d} W_{t} \\
X_{0}^{\alpha .}=x \in \bar{\Omega}
\end{array}\right.
$$

where $\alpha . \in \mathcal{A}$ is the control. There is a well-known link between the associated value function $v$ and the HJB operator $F$ given by (1.1) with $a:=2 \sigma \sigma^{T}$ (see, e.g., [23]). Using our Liouville-type result we show that $v$ is a constant that, under suitable conditions, we can explicitly compute.

The second application concerns the so-called small discount limit. Letting $\left(v_{\lambda}\right)_{\lambda>0}$ be the infinite-horizon discounted value functions:

$$
v_{\lambda}(x):=\inf _{\alpha, \in \mathcal{A}} \mathbb{E}\left[\int_{0}^{\infty} \mathrm{e}^{-\lambda t} l\left(X_{t}^{\alpha .}, \alpha_{t}\right) \mathrm{d} t\right], \quad x \in \bar{\Omega},
$$

where $X_{t}^{\alpha .}$ solves (1.5) and $l$ is bounded, we prove that $\lambda v_{\lambda}(x) \rightarrow c$ and $v_{\lambda}(x)-v_{\lambda}(\tilde{x}) \rightarrow \chi(x)$ as $\lambda \rightarrow 0$ locally uniformly, where $(c, \chi)$ solves the ergodic HJB equation (1.3) and $\tilde{x}$ is any point in $\Omega$. This is related with the ergodic control problem for stochastic processes, where $c$ is the optimal cost in the minimisation of the limit of $T^{-1} \mathbb{E}\left[\int_{0}^{T} l\left(X_{t}^{\alpha}, \alpha_{t}\right) \mathrm{d} t\right]$ as $T \rightarrow+\infty$, and $D \chi$ allows to synthesise an optimal feedback (at least in principle, under further assumptions). There is a large literature on this topic, see $[12,14,16,28]$ for diffusions reflected at the boundary and Neumann boundary conditions in (1.3), [1,3] for periodic boundary conditions, and the recent monograph [2], as well as the references therein. Some of the cited papers deal with the model problem

$$
-\Delta u+|D u|^{p}-f(x)=c, \quad \text { in } \Omega,
$$

for $p>1$, which is a special case of (1.3) with unbounded drift $b$ and running cost $l$, see, e.g., [28]. Lasry and Lions [26] showed that, in the case $1<p \leq 2$, the problem is uniquely solvable, up to constants, under the boundary condition

$$
u(x) \rightarrow+\infty \quad \text { as } x \rightarrow \partial \Omega .
$$

They use the small discount approximation (see also [30]), and show the connection of the singular boundary condition with stochastic control under state constraints. 
A related result on the small discount approximation for linear operators with singular drift was obtained in [27], where it is considered in particular the following problem

$$
\lambda u(x)-\frac{b(x)}{d(x)} \cdot D u(x)-a \Delta u-f(x)=0, \quad \text { in } \Omega,
$$

under the assumptions $\lambda>0, b(x) \cdot D d(x)>a>0$ and $b(x) \cdot \tau(x)=0$ for every $x \in \partial \Omega$ and every $\tau(x)$ tangential vector to $\partial \Omega$ at $x$. The authors prove the existence of a $C^{2}(\Omega) \cap W^{1, \infty}(\Omega)$ solution of such problem, which is the unique $C^{2}(\Omega)$ solution such that (1.2) is satisfied (see [27], Thm. 6).

Here, differently from [26-28], we make the assumption that $b(x, \alpha)$ is bounded, so $F[u]$ grows at most linearly in $D u$ and is not necessarily coercive. On the other hand we make assumptions on the coefficients $b$ and $a$ at the boundary that imply an invariance property of the domain such that the control problems of reflected diffusion and state constraint are essentially equivalent. Our weaker boundary condition (1.2) fits this context. Our assumptions on $b$ and $a$ near $\partial \Omega$ are related to the characteristic boundary points for linear operators [22] and to the irrelevant points for the generalized Dirichlet problem $[10,11]$. They also arise in the recent work [15] on the generalized principal eigenvalue and the Maximum Principle for degenerate operators such as $F$.

Let us mention two additional sources of interest for the ergodic PDE (1.3) that we do not develop here. The first is the theory of homogenisation and singular perturbations for fully nonlinear equations. In that context (1.3) is called the cell problem, the constant $c$ allows to define an effective Hamiltonian for the limit $\mathrm{PDE}$, and $\chi$ is called the corrector and is a fundamental tool for proving the convergence, see, e.g., [1] and the references therein. The second is the asymptotic behavior as $t \rightarrow+\infty$ of solutions of the evolution equation

$$
u_{t}+\sup _{\alpha \in A}\left(-b(x, \alpha) \cdot D u-\operatorname{tr}\left(a(x, \alpha) D^{2} u\right)-l(x, \alpha)\right)=0, \quad x \in \Omega, t>0,
$$

with initial datum $u(x, 0)=u_{0}(x)$. In several cases, such as periodic boundary conditions, it is known that $u(x, t) / t \rightarrow c$ as $t \rightarrow+\infty$, where $c$ is the constant solving (1.3), a fact also related to ergodic stochastic control, see, e.g., $[1,3]$. In some cases it can be proved, more precisely, that $u(x, t)-c t \rightarrow \chi(x)$, see, e.g., [13]. The validity of these results with the boundary condition (1.2) will be the subject of further investigations.

The paper is organized as follows. In Section 2 we list the precise assumptions and state the two main results of the paper. Section 3 is devoted to the construction of a strict supersolution to $F[u]=0$ with suitable boundary behavior, that plays the role of a Lyapunov function, first when $\partial \Omega$ is of class $C^{2}$ and then when it is nonsmooth under further assumptions on $F$. In Section 4 we prove the non existence of non trivial sub and supersolutions to $F[u]=0$ for $\partial \Omega$ smooth and nonsmooth. Section 5 deals with the stochastic control problem with exit times. In Section 6 we first prove the well posedness of the ergodic HJB equation (1.3) and then interpret the result within stochastic control theory as a vanishing discount limit.

\section{MAIN RESULTS}

Throughout the paper we will assume, if not otherwise stated, that $\Omega$ be a bounded connected open set in $\mathbb{R}^{n}$ with $C^{2}$ boundary. Let $d(x)$ be the signed distance function from $\partial \Omega$, i.e.

$$
d(x):=\operatorname{dist}\left(x, \mathbb{R}^{n} \backslash \Omega\right)-\operatorname{dist}(x, \bar{\Omega}) .
$$

We know, from e.g. ([24], Lem. 14.16), that $d$ is of class $C^{2}$ in some neighborhood $\overline{\Omega_{\delta}}$ of the boundary, where, here and in the sequel,

$$
\Omega_{\delta}:=\{x \in \Omega \mid d(x)<\delta\} .
$$

We consider the fully nonlinear elliptic operator (1.1), with $A \subseteq \mathbb{R}^{m}$ closed and

$$
b: \bar{\Omega} \times A \rightarrow \mathbb{R}^{n}, \quad a: \bar{\Omega} \times A \rightarrow \mathbf{M}_{n \times n}
$$


bounded and continuous, $\mathbf{M}_{n \times n}$ being the space of $n \times n$ real matrices. We further assume that $a(x, \alpha)$ is symmetric and nonnegative definite for all $x, \alpha$. This implies that $a \equiv \sigma \sigma^{T}$ for some $\sigma: \bar{\Omega} \times A \rightarrow \mathbf{M}_{n \times r}$, $r \geq 1$. Notice that we could take $\sigma \in \mathbf{M}_{n \times n}$ and symmetric, but we use this notation since in the following, for application to stochastic control problems, the matrix $a$ will be obtained from a non symmetric $\sigma$.

In the interior of $\Omega$ the diffusion is assumed to be non-degenerate. For some results, this will be required in the weak sense that the Strong Maximum Principle holds:

if $u \in U S C(\Omega)$ is a viscosity subsolution to $F[u]=0$ in $\Omega$ and there exists $x_{0} \in \Omega$ such that $u\left(x_{0}\right)=\max _{\Omega} u$, then $u$ is constant.

For a detailed analysis of this property for the HJB operator $F$ see [6]. It is satisfied if for any $x \in \Omega$ there exists $\alpha_{x} \in A$ such that the corresponding linear operator $-\operatorname{tr}\left(a\left(x, \alpha_{x}\right) D^{2} u\right)$ satisfies Hörmander hypoellipticity condition. In particular, it holds if $a\left(x, \alpha_{x}\right)>0$ in the sense of matrices.

The main regularity assumptions on the coefficients with respect to $x$ will be the following. There exists a modulus of continuity $\omega$ such that

$$
\forall x, y \in \bar{\Omega}, \alpha \in A, \quad|b(x, \alpha)-b(y, \alpha)| \leq \omega(|x-y|) .
$$

A modulus of continuity $\omega$ is a nonnegative function, continuous at 0 with $\omega(0)=0$. Moreover, we assume that the square root $\sigma$ of the matrix $a$ is Hölder or Lipschitz continuous uniformly in $\alpha$, with sufficiently large Hölder exponent, i.e.

$$
\exists \beta \in(1 / 2,1], B>0, \quad \forall x, y \in \bar{\Omega}, \alpha \in A, \quad|\sigma(x, \alpha)-\sigma(y, \alpha)| \leq B|x-y|^{\beta},
$$

where, even for matrices, $|\cdot|$ stands for the standard Euclidean norm.

Remark 2.1. Note that we are not assuming Lipschitz continuity of the coefficients of the operator $F$, but just uniform continuity of the drift and Hölder continuity of the matrix $\sigma$, which in particular assure that the operator $F$ is continuous. We will strengthen these assumptions to Hölder continuity in Section 6 and to Lipschitz continuity in applications to stochastic control problems.

The regularity assumption (2.4) is given directly on the matrix $\sigma$ as it is natural for applications to stochastic control problems. In any case, we recall some sufficient conditions that can be imposed on the matrix $a$ in order to get that (2.4) holds on the symmetric square root $\sigma$. Assume that $a(\cdot, \alpha) \in W^{2, p}(\Omega)$ for every $\alpha \in A$ with $p>1$ and that $\|a(\cdot, \alpha)\|_{W^{2, p}} \leq C$ for $C$ independent of $\alpha \in A$. Then it is proved in ([29], Thm. 1) that $\sigma(\cdot, \alpha) \in W^{1,2 p}(\Omega)$, and moreover it can be observed that the $W^{1,2 p}$ norm of $\sigma$ is independent of $\alpha$. Using Morrey's inequality we have that if $p=\infty$, then (2.4) is satisfied with $\beta=1$, and if $p>2 n$, then (2.4) is satisfied with $\beta=1-\frac{n}{p}$.

We will make different kind of assumptions about the behavior of $F$ at $\partial \Omega$. The first and weaker one is the following.

$$
\begin{aligned}
& \exists \delta, k>0, \gamma<2 \beta-1, \text { such that, for all } \bar{x} \in \partial \Omega, \text { there is } \bar{\alpha} \in A \text { for which } \\
& \left\{\begin{array}{l}
\sigma^{T}(\bar{x}, \bar{\alpha}) D d(\bar{x})=0, \\
\forall x \in \Omega \cap B_{\delta}(\bar{x}), \quad b(x, \bar{\alpha}) \cdot \operatorname{Dd}(x)+\operatorname{tr}\left(a(x, \bar{\alpha}) D^{2} d(x)\right) \geq k d^{\gamma}(x),
\end{array}\right.
\end{aligned}
$$

where $\beta$ is the exponent in (2.4). The first condition in (2.5) means that at any boundary point, the normal is a direction of degeneracy for $F$, at least for some $\alpha$. The second condition is ensured if at the boundary the normal component of the drift points inward and is sufficiently large. We will see that (2.5) ensures the existence of an appropriate Lyapunov function for the system, playing the same role as the already mentioned condition in [27]. Notice however that condition (2.5) does not prevent the function $b(\cdot, \bar{\alpha}) \cdot D d(\cdot)+\operatorname{tr}\left(a(\cdot, \bar{\alpha}) D^{2} d(\cdot)\right)$ from vanishing at $\bar{x}$, even though, in such case, it cannot be Lipschitz continuous because $\gamma<1$.

Remark 2.2. A sufficient condition for (2.5) to hold (with $\gamma=0$ ) is the following: there exists $k>0$ such that for all $\bar{x} \in \partial \Omega$ there is $\bar{\alpha} \in A$ such that the first condition in (2.5) holds and

$$
b(\bar{x}, \bar{\alpha}) \cdot D d(\bar{x})+\operatorname{tr}\left(a(\bar{x}, \bar{\alpha}) D^{2} d(\bar{x})\right)>k .
$$


Indeed, from (2.6), using the hypotheses (2.3), (2.4) together with the $C^{2}$ regularity of $d$ in a neighborhood of the compact set $\partial \Omega$, we deduce the existence of $\delta>0$ independent of $\bar{x}$ such that the second condition in $(2.5)$ holds with $\gamma=0$ and $k$ replaced by $k / 2$. Notice that this argument does not work with $k=0$, even though $\partial \Omega$ is compact, because of the first condition in (2.5). We exhibit a counter-example in Remark 3.3 below, where we do not know if the Liouville property holds.

Our first main theorem is the following result on nonexistence of nonconstant subsolutions.

Theorem 2.3. Let (2.3)-(2.5) hold and let F satisfy the Strong Maximum Principle in $\Omega$. If $u \in U S C(\Omega)$ is a viscosity subsolution to $F[u]=0$ satisfying

$$
\limsup _{x \rightarrow \partial \Omega} \frac{u(x)}{-\log \mathrm{d}(x)} \leq 0
$$

then $u$ is constant.

Remark 2.4. Observe that Theorem 2.3 does not hold without imposing some growth condition at the boundary. Indeed one can check that, in $\Omega=(-1,1)$, if $a(x) \geq d^{\ell}(x)$ for some $\ell>0$ and $d(x)$ small enough, then the function

$$
u(x)=\left|\int_{0}^{x} \mathrm{e}^{d^{-\ell}(s)} \mathrm{d} s\right|
$$

is a subsolution to $F[u]=0$ in $\Omega_{\delta}$, for $\delta$ small enough. Setting $u(x)=u(1-\delta)$ outside $\Omega \backslash \Omega_{\delta}$, one gets a subsolution to $F[u]=0$.

On the other hand, we point out that the growth condition (2.7) in Theorem 2.3 can be weakened to the following: there exists $\kappa>0$ such that

$$
\limsup _{x \rightarrow \partial \Omega} u(x) d(x)^{\kappa} \leq 0 .
$$

For more details we refer to Remark 4.3. We decided to work with the more restrictive growth condition (2.7) because this provides us with a more detailed information on the boundary behavior of the solution of the ergodic problem, c.f. Theorem 2.5.

The analogous result for supersolutions to $F[u]=0$ can be obtained under stronger assumptions. In particular we have to strengthen condition (2.5) to hold for every $\alpha \in A$, in the following sense.

$$
\begin{gathered}
\exists \delta, k>0, \gamma<2 \beta-1, \text { such that, for all } \bar{x} \in \partial \Omega \text { and for all } \alpha \in A, \\
\left\{\begin{array}{l}
\sigma^{T}(\bar{x}, \alpha) D d(\bar{x})=0, \\
\forall x \in \Omega_{\delta}, \quad b(x, \alpha) \cdot \operatorname{Dd}(x)+\operatorname{tr}\left(a(x, \alpha) D^{2} d(x)\right) \geq k d^{\gamma}(x) .
\end{array}\right.
\end{gathered}
$$

Moreover, we assume that the operator $F[u]$ satisfies the Strong Minimum Principle, that is, the operator $-F[-u]$ satisfies the Strong Maximum Principle. This property for our HJB operator $F$ was studied in [8]. A sufficient condition for it to hold true is that

$$
a(x, \alpha)>0 \text { for every } x \in \Omega \text { and } \alpha \in A \text {. }
$$

Next, we use the results on nonexistence of nontrivial solutions to derive the unique solvability of the ergodic problem (1.3) under the boundary condition (1.2). We strengthen the continuity assumption on the drift $b$ of the operator (1.1) and require the same hypothesis on the source term $l$ :

$$
\exists \gamma \in(0,1], \quad \forall x, y \in \bar{\Omega}, \alpha \in A, \quad|b(x, \alpha)-b(y, \alpha)|,|l(x, \alpha)-l(y, \alpha)| \leq B|x-y|^{\gamma} .
$$


Theorem 2.5. Under the assumptions (2.4), (2.8)-(2.10), there exists a unique $c \in \mathbb{R}$ such that the equation (1.3) admits a viscosity solution $\chi$ satisfying

$$
\lim _{x \rightarrow \partial \Omega} \frac{\chi(x)}{-\log d(x)}=0 .
$$

Moreover $\chi \in C^{2}(\Omega)$ and is unique up to addition of constants among all solutions to (1.3) which satisfy (2.11).

Actually we prove a stronger uniqueness result for $c$ and $\chi$, see Proposition 6.3.

\section{LyAPUNOV FUNCTIONS}

\subsection{The case of smooth boundary}

Proposition 3.1. Under assumptions (2.4) and (2.5), for every $M \geq 0$, there exists $\delta>0$ such that

$$
F[-\log (d(x))]>M, \quad x \in \Omega_{\delta},
$$

where $\Omega_{\delta}$ is defined in (2.2).

Proof. Take $\delta, k, \gamma$ from (2.5). Up to reducing $\delta$ if needed, we can suppose that $d \in C^{2}\left(\overline{\Omega_{\delta}}\right)$. In particular, for given $x \in \Omega_{\delta}$, we can consider its unique projection on $\partial \Omega$, i.e., the point $\bar{x} \in \partial \Omega$ satisfying $|x-\bar{x}|=d(x)<\delta$. Let $\bar{\alpha}$ be such that (2.5) is verified. The first condition in (2.5) and property (2.4) yield

$$
\left|\sigma^{T}(x, \bar{\alpha}) D d(x)\right|=\left|\sigma^{T}(x, \bar{\alpha}) D d(\bar{x})\right| \leq|\sigma(x, \bar{\alpha})-\sigma(\bar{x}, \bar{\alpha})| \leq B d^{\beta}(x) .
$$

Therefore we get

$$
\begin{aligned}
F[-\log d(x)] & =\sup _{\alpha \in A}\left(b(x, \alpha) \cdot \frac{D d(x)}{d(x)}+\operatorname{tr}\left(a(x, \alpha) \frac{D^{2} d(x)}{d(x)}\right)-\frac{1}{d^{2}(x)}\left|\sigma^{T}(x, \alpha) D d(x)\right|^{2}\right) \\
& \geq \frac{1}{d(x)}\left(b(x, \bar{\alpha}) \cdot D d(x)+\operatorname{tr}\left(a(x, \bar{\alpha}) D^{2} d(x)\right)-\frac{1}{d^{2}(x)}\left|\sigma^{T}(x, \bar{\alpha}) D d(x)\right|^{2}\right. \\
& \geq k d^{\gamma-1}(x)-B^{2} d^{2 \beta-2}(x) .
\end{aligned}
$$

Since $\gamma-1<2 \beta-2$, we can further reduce $\delta$, independently of $x$, in such a way that $k d^{\gamma-1}-B^{2} d^{2 \beta-2}>M$ in $\Omega_{\delta}$, concluding the proof of the proposition.

If we replace (2.5) with (2.8), then the following stronger version of Proposition 3.1 holds.

Proposition 3.2. Let (2.4) and (2.8) hold. Then for every $M \geq 0$ there exists $\delta>0$ such that

$$
F[\log d(x)]<-M, \quad x \in \Omega_{\delta} .
$$

Proof. By explicit computation,

$$
F[\log d(x)]=-\inf _{\alpha \in A}\left(b(x, \alpha) \cdot \frac{D d(x)}{d(x)}+\operatorname{tr}\left(a(x, \alpha) \frac{D^{2} d(x)}{d(x)}\right)-\frac{1}{d^{2}(x)}\left|\sigma^{T}(x, \alpha) D d(x)\right|^{2}\right) .
$$

The conclusion then follows from a straightforward adaptation of the argument in the proof of Proposition 3.1.

We conclude this section with two comments about the optimality of condition (2.5) for the construction of the Lyapunov function. 
Remark 3.3. The function $-\log d(x)$ might not be a Lyapunov function if (2.5) is relaxed to the following: for all $\bar{x} \in \partial \Omega$ there is $\bar{\alpha} \in A$ for which

$$
\sigma^{T}(\bar{x}, \bar{\alpha}) D d(\bar{x})=0, \quad b(\bar{x}, \bar{\alpha}) \cdot D d(\bar{x})+\operatorname{tr}\left(a(\bar{x}, \bar{\alpha}) D^{2} d(\bar{x})\right)>0 .
$$

Let us show this with an example. Let $\Omega$ be a domain in $\mathbb{R}^{2}$ coinciding, in a neighborhood of $(0,0) \in \partial \Omega$, with the half-plane $\{(x, y), x \in \mathbb{R}, y>0\}$. Take $A=\{1,2\}$,

$$
\begin{aligned}
& b(x, y, 1)=(0,1), \quad \sigma(x, y, 1)=\left(\begin{array}{cc}
1 & 0 \\
0 & x^{2}+y
\end{array}\right), \\
& b(x, y, 2)=\left(0, x^{4}-y\right), \quad \sigma(x, y, 2)=\left(\begin{array}{ll}
y & 0 \\
0 & y
\end{array}\right),
\end{aligned}
$$

and $a=\sigma \sigma^{T}$. Note that in a neighborhood of $(0,0), d(x, y)=y$, whence $\operatorname{Dd}(x, y)=(0,1)$ and $D^{2} d(x, y)=0$. One can readily check that (3.2) holds at the points $(x, 0)$, with $\bar{\alpha}=1$ if $x=0$ and $\bar{\alpha}=2$ otherwise, whereas the second condition in (2.5) is not verified at $\bar{x}=(0,0)$ by neither $\bar{\alpha}=1$ nor $\bar{\alpha}=2$. Let us check that $-\log d(x)$ is not a supersolution of $F=0$ in a neighborhood of $(0,0)$. For $(x, y) \in \Omega$ close to $(0,0)$, we have that

$$
\begin{aligned}
F[-\log d(x)] & =\max _{\alpha \in\{0,1\}}\left(b(x, \alpha) \cdot \frac{D d(x)}{d(x)}+\operatorname{tr}\left(a(x, \alpha) \frac{D^{2} d(x)}{d(x)}\right)-\frac{1}{d^{2}(x)}\left|\sigma^{T}(x, \alpha) D d(x)\right|^{2}\right) \\
& =\max \left\{\frac{1}{y}-\frac{\left(x^{2}+y\right)^{2}}{y^{2}}, \frac{x^{4}}{y}-2\right\} .
\end{aligned}
$$

Now, if $y=x^{4}$, then

$$
\frac{x^{4}}{y}-2=-1<0, \quad \frac{1}{y}-\frac{\left(x^{2}+y\right)^{2}}{y^{2}}=\frac{1}{y}-\frac{(\sqrt{y}+y)^{2}}{y^{2}}<0 .
$$

This shows that $F[-\log d(x)]<0$ at some points arbitrarily close to $(0,0)$.

Remark 3.4. Note that $\gamma$ in (2.5) satisfies $\gamma<1$, because $\beta \leq 1$. If we allow $\gamma=1$, then we are only able to obtain a "weak" Lyapunov function, that is a strict supersolution to the equation in a neighborhood of the boundary, but not exploding at the boundary. Indeed if we assume that the second condition in (2.5) holds with $\gamma=1$, then for every $\eta>0$, there exists $\delta>0$ such that

$$
F[-\log (d(x)+\eta)]>0, \quad x \in \Omega_{\delta} .
$$

This follows from the same arguments as in the proof of Proposition 3.1, the final step being now the choice of $\delta>0$ such that

$$
\forall x \in \Omega_{\delta}, \quad \frac{k d(x)}{d(x)+\eta}-B^{2} \frac{d^{2 \beta}(x)}{(d(x)+\eta)^{2}}>0 .
$$

\subsection{Case of non-smooth boundary}

When the domain $\Omega$ is not assumed to be $C^{2}$, a partial result in the direction of Proposition 3.1 is obtained under the following assumption: for all $\bar{x} \in \partial \Omega$, there exist $\bar{\alpha} \in A$, a neighborhood $U$ of $\bar{x}$ and a constant $k>0$ such that

$$
-b(x, \bar{\alpha}) \cdot D d(x)-\operatorname{tr} a(x, \bar{\alpha}) D^{2} d(x) \leq-k, \quad x \in \Omega \cap U
$$

in viscosity sense, and

$$
\forall z \in \partial \Omega \cap U, \quad \forall \nu \in N(z), \quad \sigma^{T}(z, \bar{\alpha}) \nu=0,
$$

where $N(z)$ is the interior normal cone to $\Omega$ at $z$, or, equivalently, the set of generalized exterior normals to $\Omega^{c}$ as defined in ([4], p. 48). Namely, a unit vector $\nu$ belongs to $N(z), z \in \partial \Omega$, if there exists $x \in \Omega$ such that 
$x=z+d(x) \nu$. Due to the possible lack of regularity of $d$, condition (3.3) has to be understood in the following sense:

$$
\forall x \in \Omega \cap U, \quad \forall(p, Y) \in J^{2,+} d(x), \quad-b(x, \bar{\alpha}) \cdot p-\operatorname{tr} a(x, \bar{\alpha}) Y \leq-k,
$$

where

$$
J^{2,+} d(x):=\left\{(p, Y) \in \mathbb{R}^{n} \times \mathbf{S}_{n} \mid \limsup _{\bar{\Omega} \ni y \rightarrow x} \frac{\mathrm{d}(y)-d(x)-p \cdot(y-x)-\frac{1}{2}(y-x) Y \cdot(y-x)}{|y-x|^{2}} \leq 0\right\} .
$$

If the boundary is smooth, (3.3) is equivalent to (2.6) whereas (3.4) is slightly stronger than the first condition in (2.5), so, combined, they imply condition (2.5) (see Rem. 2.2).

The normal cone $N(z)$ and the set of projections

$$
P(x):=\{z \in \partial \Omega:|x-z|=d(x)\}
$$

allow us to give the following representation.

Lemma 3.5. For $x \in \Omega$, let $(p, Y) \in J^{2,+} d(x)$. Then $p=\lim _{j} p_{j}$ where for all $j$ there exist $\lambda_{i} \geq 0, i=1, \ldots, N$, $\sum_{i=1}^{N} \lambda_{i}=1$, and $z_{i} \in P(x)$ such that

$$
p_{j}=\sum_{i=1}^{N} \lambda_{i} \nu_{i}, \quad \nu_{i} \in N\left(z_{i}\right)
$$

Proof. The condition $(p, Y) \in J^{2,+} d(x)$ yields

$$
p \in D^{+} d(x):=\left\{q \in \mathbb{R}^{n} \mid \limsup _{\bar{\Omega} \ni y \rightarrow x} \frac{\mathrm{d}(y)-d(x)-q \cdot(y-x)}{|y-x|} \leq 0\right\} .
$$

Proposition II.2.14 in [4] states that the set $D^{+} d(x)$ coincides with the closure of the convex hull of the set

$$
\left\{\frac{x-z}{|x-z|}: z \in P(x)\right\} \text {. }
$$

On the other hand, $z \in P(x)$ implies $x=z+d(x) \frac{x-z}{|x-z|}$ and therefore $\frac{x-z}{|x-z|} \in N(z)$.

Proposition 3.6. Let (2.3), (2.4), (3.3), (3.4) hold. Then, for any $M \geq 0$, there exists $\delta>0$ such that $-\log (d(x))$ satisfies in viscosity sense

$$
F\left[-\log (d(x)]>M, \quad x \in \Omega_{\delta} .\right.
$$

Proof. Note that (2.3), (2.4) assure that the operator $F[u]$ is continuous. For a fixed $\bar{x} \in \partial \Omega$ take $\bar{\alpha} \in A$ and the neighborhood $U$ such that (3.3) and (3.4) hold. For $x \in \Omega \cap U$ consider $(p, Y) \in J^{2,+} d(x)$. Take $p_{j} \rightarrow p$ as in Lemma 3.5. The orthogonality condition (3.4) yields

$$
\sigma^{T}(x, \bar{\alpha}) p_{j}=\sum_{i=1}^{N} \lambda_{i} \sigma^{T}(x, \bar{\alpha}) \nu_{i}=\sum_{i=1}^{N} \lambda_{i}\left(\sigma^{T}(x, \bar{\alpha})-\sigma^{T}\left(z_{i}, \bar{\alpha}\right)\right) \nu_{i}
$$

Then, by (2.4),

$$
\left|\sigma^{T}(x, \bar{\alpha}) p_{j}\right| \leq \sum_{i=1}^{N} \lambda_{i} B\left|x-z_{i}\right|^{\beta}=B d^{\beta}(x)
$$


from which, letting $j \rightarrow \infty$,

$$
\left|\sigma^{T}(x, \bar{\alpha}) p\right| \leq B d^{\beta}(x) .
$$

Now we want to prove that $F[-\log d(x)]>0$ in viscosity sense. Note that if $(q, Z) \in J^{2,-}(-\log d(x))$ then there exist $(p, Y) \in J^{2,+} d(x)$ such that $q=-\frac{p}{d(x)}$ and $Z=-\frac{Y}{d(x)}+\frac{p \otimes p}{d^{2}(x)}$. Using (3.5) we derive

$$
\begin{aligned}
F(x, p, Y) & =\sup _{\alpha \in A}\left(b(x, \alpha) \cdot \frac{p}{d(x)}+\operatorname{tr} a(x, \alpha) \frac{Y}{d(x)}-\frac{1}{d^{2}(x)}\left|\sigma^{T}(x, \alpha) p\right|^{2}\right) \\
& \geq \frac{1}{d(x)}\left(b(x, \bar{\alpha}) \cdot p+\operatorname{tr}(a(x, \bar{\alpha}) Y)-\frac{1}{d^{2}(x)}\left|\sigma^{T}(x, \bar{\alpha}) p\right|^{2}\right. \\
& \geq \frac{1}{d(x)}\left(k-B^{2} d^{2 \beta-1}(x)\right) .
\end{aligned}
$$

So, for given $M>0, \tilde{\delta}>0$ can be chosen sufficiently small, depending on $\bar{x}$ (because $k$ does) but independent of $x, p, Y$, in such a way that

$$
F[-\log d(x)]>M, \quad x \in \Omega_{\tilde{\delta}} \cap U
$$

in viscosity sense. This means that there is a neighborhood $W$ of $\partial \Omega$ such that

$$
F[-\log d(x)]>M, \quad x \in \Omega \cap W,
$$

and thus the conclusion of the proposition holds for some $\delta>0$ by the compactness of $\partial \Omega$.

Remark 3.7. If we assume in Proposition 3.6 that conditions (3.3) and (3.4) hold for every $\alpha \in A$, then we obtain that for any $M \geq 0$, there exists $\delta>0$ such that $-\log (d(x))$ satisfies in viscosity sense

$$
\inf _{\alpha \in A}\left(-b(x, \alpha) \cdot D(-\log d(x))-\operatorname{tr}\left(a(x, \alpha) D^{2}(-\log d(x))\right)\right)>M, \quad x \in \Omega_{\delta} .
$$

\section{Nonexistence of nONCONSTANT SOlutions}

Using the Lyapunov functions constructed in the previous section we now prove that $F[u]=0$ has only trivial sub and supersolutions.

Proof of Theorem 2.3. Let $\delta$ be from Proposition 3.1 corresponding to $M=0$. We can assume without loss of generality that $\delta<1$. Define $\Omega_{\delta}$ as in $(2.2)$ and

$$
K_{\delta}:=\max _{\Omega \backslash \Omega_{\delta}} u .
$$

For $\varepsilon>0$ the function $u(x)-\left(K_{\delta}-\varepsilon \log d(x)\right)$ is negative when $d(x)=\delta$ and, by (2.7), it goes to $-\infty$ as $x \rightarrow \partial \Omega$. Suppose by contradiction that it is positive somewhere in $\Omega_{\delta}$. Then there exists $x_{0} \in \Omega_{\delta}$ such that

$$
u\left(x_{0}\right)-\left(K_{\delta}-\varepsilon \log d\left(x_{0}\right)\right)=\max _{x \in \Omega_{\delta}}\left[u(x)-\left(K_{\delta}-\varepsilon \log d(x)\right)\right] .
$$

Since $u$ is a subsolution to $F[u]=0$, this implies that

$$
\varepsilon F\left[-\log d\left(x_{0}\right)\right] \leq 0,
$$

which is impossible by Proposition 3.1. We have therefore shown that

$$
\forall x \in \Omega_{\delta}, \quad u(x) \leq K_{\delta}-\varepsilon \log d(x),
$$

whence, letting $\varepsilon \rightarrow 0, u \leq K_{\delta}$ in $\Omega_{\delta}$. This means that $u$ achieves its maximum $K_{\delta}$ inside $\Omega$ and then it is constant since $F$ satisfies the Strong Maximum Principle in $\Omega$. 
In the case of nonsmooth boundary, the same result holds true, under stronger regularity assumptions on the coefficients.

Proposition 4.1. Instead of $\partial \Omega$ of class $C^{2}$ assume that any $\bar{x} \in \partial \Omega$ has a a neighborhood $U$ such that (3.3) and (3.4) hold for some $\bar{\alpha} \in A$ and $k>0$. Suppose also that $b, \sigma$ are Lipschitz continuous, i.e., the continuity modulus in (2.3) is $\omega(r)=B r$ for some $B>0$ and $\beta=1$ in (2.4), and $F$ satisfies the Strong Maximum Principle in $\Omega$. If $u \in U S C(\Omega)$ is a viscosity subsolution to $F[u]=0$ satisfying $(2.7)$ then $u$ is constant.

Proof. We use the same notation as in Theorem 2.3. Now, owing to Proposition 3.6, $0<\delta<1$ is chosen in such a way that $-\log d(x)$ satisfies in viscosity sense $F[-\log d(x)]>1$ in $\Omega_{\delta}$. Using the doubling variables method we define, for $\eta>0$ and $x, y \in \Omega_{\delta}$,

$$
\Phi(x, y):=u(x)-K_{\delta}+\varepsilon \log \mathrm{d}(y)-\frac{|x-y|^{2}}{2 \eta} .
$$

Observe that, due to $(2.7), \sup _{\left(\Omega_{\delta}\right)^{2}} \Phi<+\infty$. Take $\left(x_{\eta}, y_{\eta}\right)$ small such that $\Phi\left(x_{\eta}, y_{\eta}\right)=\sup _{\Omega_{\delta}^{2}} \Phi$. By $([21]$, Lem. 3.1), $\frac{\left|x_{\eta}-y_{\eta}\right|^{2}}{\eta} \rightarrow 0$ as $\eta \rightarrow 0$, and up to subsequences, $x_{\eta}, y_{\eta} \rightarrow x \in \Omega_{\delta}$ such that $u(x)-K_{\delta}+\varepsilon \log d(x)=$ $\sup _{\Omega_{\delta}} u-K_{\delta}+\varepsilon \log d$.

If $\Phi\left(x_{\eta}, y_{\eta}\right) \leq 0$ then $\Phi(x, x) \leq 0$ for $x \in \Omega_{\delta}$, from which, arguing as in the proof of Theorem 2.3, we infer that $u$ achieves a maximum in $\Omega$ and then it is constant.

Suppose then that $\Phi\left(x_{\eta}, y_{\eta}\right)>0$ for all $\eta>0$. By classical argument (see [21], Thm. 3.2), we get that there exist $X, Y \in \mathbf{S}_{n}$ such that $\left(\frac{x_{\eta}-y_{\eta}}{\eta}, X\right) \in J^{2,+} u\left(x_{\eta}\right),\left(\frac{x_{\eta}-y_{\eta}}{\eta}, Y\right) \in J^{2,-}\left(-\varepsilon \log d\left(y_{\eta}\right)\right)$ and

$$
p X \cdot p-q Y \cdot q \leq \frac{1}{\eta}|p-q|^{2} \quad \forall p, q \in \mathbb{R}^{n} .
$$

Let $e_{i}$, for $i=1, \ldots, r$ the $i$-th unit vector. Then it is easy to check for every $\alpha \in A, \operatorname{tr} a\left(x_{\eta}, \alpha\right) X=$ $\sum_{i=1}^{r}\left(\sigma\left(x_{\eta}, \alpha\right) e_{i}\right) X \cdot\left(\sigma\left(x_{\eta}, \alpha\right) e_{i}\right)$. So, by (4.1) applied to $p=\sigma\left(x_{\eta}, \alpha\right) e_{i}, q=\sigma\left(y_{\eta}, \alpha\right) e_{i}$, we get that for every $\alpha \in A$,

$$
\operatorname{tr} a\left(x_{\eta}, \alpha\right) X-\operatorname{tr} a\left(y_{\eta}, \alpha\right) Y \leq \frac{1}{\eta} \sum_{i=1}^{r} \sum_{j=1}^{n}\left|\sigma_{j i}\left(x_{\eta}, \alpha\right)-\sigma_{j i}\left(y_{\eta}, \alpha\right)\right|^{2} \leq B^{2} \frac{\left|x_{\eta}-y_{\eta}\right|^{2}}{\eta},
$$

where we used the fact that $\sigma$ is Lischitz continuous. Using the fact that $b$ is Lipschitz continuous we get

$$
\left|b\left(y_{\eta}, \alpha\right)-b\left(x_{\eta}, \alpha\right)\right| \frac{\left|x_{\eta}-y_{\eta}\right|}{\eta} \leq B\left(\frac{\left|x_{\eta}-y_{\eta}\right|^{2}}{\eta}\right)
$$

Using the previous inequalities and the fact that $u$ is a subsolution to $F[u]=0$, we get

$$
\begin{aligned}
0 & \geq \varepsilon \sup _{\alpha \in A}\left(-b\left(x_{\eta}, \alpha\right) \cdot \frac{x_{\eta}-y_{\eta}}{\eta}-\operatorname{tr} a\left(x_{\eta}, \alpha\right) X\right) \\
& \geq \varepsilon \sup _{\alpha \in A}\left(-b\left(y_{\eta}, \alpha\right) \cdot \frac{x_{\eta}-y_{\eta}}{\eta}-\operatorname{tr}\left(a\left(y_{\eta}, \alpha\right) Y\right)\right)-B \varepsilon \frac{\left|x_{\eta}-y_{\eta}\right|^{2}}{\eta}-\varepsilon B^{2}\left(\frac{\left|x_{\eta}-y_{\eta}\right|^{2}}{\eta}\right),
\end{aligned}
$$

which gives, for $\eta$ sufficiently small, a contradiction to the fact that $F[-\log d(x)]>1$ in viscosity sense in $\Omega_{\delta}$.

If we strengthen condition (2.5) to condition (2.8), and let $F[u]$ satisfies the Strong Minimum Principle in $\Omega$, that is $-F[-u]$ satisfies the Strong Maximum Principle in $\Omega$, we also obtain the statement for supersolutions. 
Theorem 4.2. Let conditions (2.3), (2.4), (2.8) hold and let F satisfies the Strong Minimum Principle in $\Omega$. If $v \in L S C(\Omega)$ is a viscosity supersolution to $F[v]=0$ satisfying

$$
\liminf _{x \rightarrow \partial \Omega} \frac{v(x)}{-\log d(x)} \geq 0
$$

then $v$ is constant.

Proof. Note that $v$ is a subsolution to $-F[-u]=0$. So, we conclude, using the same arguments as in the proof of Theorem 2.3, by substituting Proposition 3.1 with Proposition 3.2.

Remark 4.3. Theorems 2.3 and 4.2 can be improved by replacing the growth conditions (2.7) and (4.2) with, respectively,

$$
\limsup _{x \rightarrow \partial \Omega} u(x) d(x)^{\kappa} \leq 0, \quad \text { and } \quad \liminf _{x \rightarrow \partial \Omega} v(x) d(x)^{\kappa} \geq 0
$$

for some $\kappa>0$.

Indeed, observe that for every $M>0$ and every $\kappa>0$, there exists $\delta>0$ such that

$$
\begin{aligned}
F\left[d(x)^{-\kappa}\right] & =\frac{\kappa}{d(x)^{\kappa}} \sup _{\alpha \in A}\left(b(x, \alpha) \cdot \frac{D d(x)}{d(x)}+\operatorname{tr}\left(a(x, \alpha) \frac{D^{2} d(x)}{d(x)}\right)-\frac{\kappa+1}{d^{2}(x)}\left|\sigma^{T}(x, \alpha) D d(x)\right|^{2}\right) \\
& \geq k \kappa d^{\gamma-1-\kappa}(x)-B^{2} \kappa(\kappa+1) d^{2 \beta-2-\kappa}(x) \geq M
\end{aligned}
$$

for every $x \in \Omega_{\delta}$, by assumption (2.5). Analogoulsy we get that for every $M>0$ and every $\kappa>0$ there exists $\delta>0$ such that $F\left[-d(x)^{-\kappa}\right] \leq-M$ for every $x \in \Omega_{\delta}$.

So, the same arguments of the proofs of Theorems 2.3 and 4.2 can be repeated by simply substituting $d(x)^{-\kappa}$ to $-\log (d(x))$.

We state the analogous of Theorem 2.3 in the case of nonsmooth boundary.

Proposition 4.4. Instead of $\partial \Omega$ of class $C^{2}$ assume that any $\bar{x} \in \partial \Omega$ has a a neighborhood $U$ such that (3.3) and (3.4) hold for every $\alpha \in A$ and $k>0$. Suppose also that $b, \sigma$ are Lipschitz continuous, i.e., the continuity modulus in (2.3) is $\omega(r)=B r$ for some $B>0$ and $\beta=1$ in (2.4), and $F$ satisfies the Strong Minimum Principle in $\Omega$. If $v \in L S C(\Omega)$ is a viscosity supersolution to $F[v]=0$ satisfying (4.2) then $v$ is constant.

Proof. Observe that $-v$ is a viscosity subsolution to

$$
\inf _{\alpha \in A}\left(-b(x, \alpha) \cdot D u-\operatorname{tr}\left(a(x, \alpha) D^{2} u\right)\right)=0
$$

and satisfies (2.7). Moreover by assumption, the operator

$$
G[u]=\inf _{\alpha \in A}\left(-b(x, \alpha) \cdot D u-\operatorname{tr}\left(a(x, \alpha) D^{2} u\right)\right)
$$

satisfies the Strong Maximum Principle. So, recalling Remark 3.7, we can repeat the proof of Proposition 4.1 by substituting the operator $F[u]$ with the operator $G[u]$.

\section{An application to stochastic CONTrol Problems With exit times}

We consider the control system (1.5), where $W_{t}$ is a $r$-dimensional standard Brownian motion and the control $\alpha$. : $\mathbb{R}_{+} \rightarrow A$ belongs to the set of admissible controls $\mathcal{A}$, namely, progressively measurable processes, with respect to the filtration associated to the Brownian motion. 
In order to have existence and uniqueness of solutions to the control system, throughout this section we will strenghten the regularity assumptions on the coefficients to the following. We assume there exists $B>0$ for which

$$
\forall x, y \in \bar{\Omega}, \alpha \in A, \quad|b(x, \alpha)-b(y, \alpha)| \leq B|x-y|, \quad|\sigma(x, \alpha)-\sigma(y, \alpha)| \leq B|x-y| .
$$

Moreover, to ensure existence of optimal controls for the optimal control problems we are going to consider, we assume that the set $A$ is compact and

$$
\{(b(x, \alpha), a(x, \alpha)) \mid \alpha \in A\} \text { is convex for all } x \in \bar{\Omega} .
$$

Define for every $x \in \Omega$ the exit time from the open set $\Omega$

$$
\tau_{x}^{\alpha .}=\inf \left\{t \geq 0 \mid X_{t}^{\alpha .} \notin \Omega\right\} \in \mathbb{R} \cup\{+\infty\} .
$$

It has been proved in [7] that under the previous assumptions, the set $\bar{\Omega}$ is viable or weakly invariant for the control system (1.5) in the following sense: for every $x \in \bar{\Omega}$ there exists an admissible control $\alpha$. such that $X_{t}^{\alpha .} \in \bar{\Omega}$ almost surely for all $t \geq 0$. In the next proposition we prove that actually in our case this result can be improved to get also the viability of the open set $\Omega$. Such a result was obtained in [17] for stochastic systems without control.

Proposition 5.1. Let (2.5), (5.1), (5.2) hold. Then for every $x \in \Omega$ there exists an admissible control $\alpha . \in \mathcal{A}$ such that $X_{t}^{\alpha .} \in \Omega$ almost surely for all $t \geq 0$, i.e. $\tau_{x}^{\alpha .}=+\infty$ almost surely.

Proof. Let $V$ be a $C^{2}$ extension of the function $-\log (d(x))$ to the whole $\Omega$, which coincides with $-\log d(x)$ in a neighborhood of $\partial \Omega$. We can assume that $V \geq 1$ in $\Omega$ and moreover, by Proposition 3.1, there exists a constant $C \geq 0$ such that

$$
F[V](x) \geq-C \quad x \in \Omega .
$$

Define for every $\delta>0$, and $x \in \Omega$, the exit time

$$
\tau_{x}^{\delta, \alpha .}=\inf \left\{t \geq 0 \mid X_{t}^{\alpha .} \notin \Omega \backslash \overline{\Omega_{\delta}}\right\} \in \mathbb{R} \cup\{+\infty\} .
$$

By superoptimality principles for viscosity solutions (see [19], Cor. 6), we get for every $\delta>0$, every $x \in \Omega \backslash \Omega_{\delta}$ and every $t \geq 0$, recalling that $V \geq 1$,

$$
V(x) \geq \inf _{\alpha, \in \mathcal{A}} \mathbb{E}\left[V\left(X_{\tau_{x}^{\delta, \alpha \cdot \wedge t}}^{\alpha \cdot}\right)-C\left(\tau_{x}^{\delta, \alpha \cdot} \wedge t\right)\right] \geq \inf _{\alpha, \in \mathcal{A}}\left[\int_{\left\{\omega \mid \tau_{x}^{\delta, \alpha \cdot}(\omega) \leq t\right\}} V\left(X_{\tau^{\delta, \alpha .}}^{\alpha \cdot}(\omega)\right) d \mathbb{P}(\omega)-C t\right],
$$

where $\tau_{x}^{\delta, \alpha \cdot} \wedge t=\min \left\{\tau_{x}^{\delta, \alpha .}, t\right\}$. Take $\delta>0$ such that $V(x)=-\log d(x)$ for $x \in \overline{\Omega_{\delta}} \backslash \partial \Omega$. From (5.4) we get that for every $t \geq 0$

$$
\inf _{\alpha . \in \mathcal{A}} \mathbb{P}\left(\omega \mid \tau_{x}^{\delta, \alpha \cdot}(\omega) \leq t\right) \leq \frac{V(x)+C t}{-\log \delta}
$$

Moreover, since $\inf _{\alpha .} \mathbb{P}\left(\omega \mid \tau_{x}^{\delta, \alpha .}(\omega) \leq t\right)$ is decreasing as $\delta \rightarrow 0$, we get that, for all $x \in \Omega$,

$$
\inf _{\delta} \inf _{\alpha \cdot \in \mathcal{A}} \mathbb{P}\left(\omega \mid \tau_{x}^{\delta, \alpha .}(\omega) \leq t\right)=0 .
$$

So, for every $t \geq 0$,

$$
\inf _{\alpha . \in \mathcal{A}} \mathbb{P}\left(\omega \mid \tau_{x}^{\alpha .}(\omega) \leq t\right)=0 .
$$

Finally we claim that (5.5) implies that

$$
\inf _{\alpha . \in \mathcal{A}} \mathbb{P}\left(\omega \mid \tau_{x}^{\alpha .}(\omega)<+\infty\right)=0 .
$$


Let $h$ be a bounded uniformly continuous function such that $h \equiv 0$ in $\Omega$ and $h>0$ in $\mathbb{R}^{n} \backslash \Omega$. Let

$$
w(x)=\inf _{\alpha \cdot \in \mathcal{A}} \mathbb{E} \int_{0}^{+\infty} h\left(X_{t}^{\alpha \cdot}\right) \mathrm{e}^{-\nu t} \mathrm{~d} t
$$

So $0 \leq w \leq \frac{\|h\|_{\infty}}{\nu}$. Moreover, by standard dynamic programming principle (see [25]) for every $t>0$

$$
w(x)=\inf _{\alpha \cdot \in \mathcal{A}} \mathbb{E}\left(w\left(X_{t}^{\alpha \cdot}\right) \mathrm{e}^{-\nu t}+\int_{0}^{t} h\left(X_{s}^{\alpha \cdot}\right) \mathrm{e}^{-\nu s} \mathrm{~d} s\right) .
$$

Fix $\varepsilon>0$ and take $t$ such that $\frac{\|h\|_{\infty}}{\nu} \mathrm{e}^{-\nu t} \leq \varepsilon$. So, for every $x \in \Omega$, due to (5.5),

$$
w(x) \leq \inf _{\alpha \cdot \in \mathcal{A}} \mathbb{E} \int_{0}^{t} h\left(X_{s}^{\alpha \cdot}\right) \mathrm{e}^{-\nu s} \mathrm{~d} s+\varepsilon \leq \varepsilon
$$

from which we deduce by arbitrariness of $\varepsilon, w \equiv 0$. This implies (5.6), recalling that under assumption (5.2), for every initial data $x \in \Omega$ there exists an optimal control for the control problem (5.7) (see [25]).

Consider a terminal cost $\phi \in C(\partial \Omega)$ that the controller pays as the system hits the boundary. We introduce the cost functional

$$
G(x, \alpha, \omega)= \begin{cases}\phi\left(X_{\tau_{x}^{\alpha}}^{\alpha \cdot}(\omega)\right) & \tau_{x}^{\alpha .}(\omega)<+\infty \\ 0 & \tau_{x}^{\alpha \cdot}(\omega)=+\infty\end{cases}
$$

and define the value function

$$
v(x)=\inf _{\alpha . \in \mathcal{A}} \mathbb{E}[G(x, \alpha ., \omega)] .
$$

We make a non degeneracy assumption on the system at a minimal point for $\phi$ which is somehow opposite to condition (2.5).

$$
\left\{\begin{array}{l}
\exists \underline{x} \in \partial \Omega, \underline{\alpha} \in A \text { such that } \phi(\underline{x})=\min \phi \text { and } \\
\text { either } \sigma^{T}(\underline{x}, \underline{\alpha}) D d(\underline{x}) \neq 0, \text { or } b(\underline{x}, \underline{\alpha}) \cdot \operatorname{Dd}(\underline{x})+\operatorname{tr}\left(a(\underline{x}, \underline{\alpha}) D^{2} d(\underline{x})\right)<0 .
\end{array}\right.
$$

Theorem 5.2. Let (2.5), (5.1), (5.2) and (5.8) hold. Then the value function $v$ satisfies

$$
\forall x \in \Omega, \quad v(x)=\min \{\min \phi, 0\} .
$$

Proof. The value function $v$ is known to satisfy a dynamic programming principle (see [25]). From this it is possible to deduce (see [10], Thm. 4.4, [11]) that the upper semicontinuous envelope of $v$, defined as

$$
v^{*}(x):=\limsup _{\bar{\Omega} \ni y \rightarrow x} v(y), \quad x \in \bar{\Omega},
$$

is a viscosity subsolution of the HJB equation $F[u]=0$ in $\Omega$ and satisfies the Dirichlet boundary condition $u \leq \phi$ on $\partial \Omega$ in viscosity sense (that we will recall later). Then, by Theorem $2.3, v^{*}$ is constant in $\Omega$, say $v^{*} \equiv c$ in $\Omega$.

By Proposition 5.1, for every $x \in \Omega$ there exists an admissible control $\alpha$ such that $X_{t}^{\alpha} \in \Omega$ almost surely for all $t \geq 0$. So, if $\min \phi>0$, then it is immediate to deduce by the definition of $v$ that $v \equiv 0$ in $\Omega$.

We assume now that $\min \phi \leq 0$, and we show that in this case $v \equiv \min \phi$ on $\Omega$. Being upper semicontinuous, $v^{*}$ satisfies $v^{*}(x) \geq c$ for $x \in \partial \Omega$. Since $v^{*} \geq v \geq \min \phi=: m$, we must prove that $m \geq c$. Assumption (5.8) allows us to build an upper barrier at the point $\underline{x}$, namely, a function $W \in C^{2}(B(\underline{x}, r))$, with $r>0$, such that:

(i) $W \geq 0$ and $L W>0$ in $B(\underline{x}, r) \cap \bar{\Omega}$, 
(ii) $W(\underline{x})=0, W(x) \geq \mu>0$ for all $x \in \bar{\Omega}$ with $|x-\underline{x}|=r$.

For $k, \lambda>0$ set

$$
W(x):=1-\mathrm{e}^{-k\left(d(x)+\lambda|x-\underline{x}|^{2}\right)} .
$$

It is easy to compute

$$
\begin{aligned}
\left(-\operatorname{tr}\left(a(\underline{x}, \alpha) D^{2} W(\underline{x})\right)\right. & -b(\underline{x}, \alpha) \cdot D W(\underline{x})) \mathrm{e}^{k\left(d(x)+\lambda|x-\underline{x}|^{2}\right)}= \\
& -k \operatorname{tr}\left(a(\underline{x}, \alpha) D^{2} d(\underline{x})\right)+k^{2}|\sigma(\underline{x}, \alpha) D d(\underline{x})|^{2}-k b(\underline{x}, \alpha) \cdot D d(\underline{x})-2 k \lambda \operatorname{tr} a(\underline{x}, \alpha) .
\end{aligned}
$$

Next we choose $\alpha=\underline{\alpha}$ and assume first the first condition in (5.8) to hold. In this case, since the coefficients are continuous and $\bar{d}$ is $C^{2}$ close to $\partial \Omega$, we can get $F[W]>0$ in a neighborhood of $\underline{x}$ by taking $k$ large enough. If, instead, the second condition in (5.8) holds, we choose $\lambda$ small to reach the same conclusion. Properties ii) of the barrier are obvious.

Assume by contradiction that $m<c$ and fix $m^{\prime} \in(m, c)$ and $r^{\prime}>0$ such that

$$
\forall x \in B\left(\underline{x}, r^{\prime}\right) \cap \partial \Omega, \quad \phi(x) \leq m^{\prime}<c .
$$

Now call $\rho:=\min \left(r, r^{\prime}\right)$ and define, for $k>0$,

$$
w(x):=k W(x)+m .
$$

Observe that $F[w]>0$ in $B(\underline{x}, \rho) \cap \bar{\Omega}$ by the homogeneity of the operator $F$. Choose also $k$ large enough so that

$$
\forall x \in \bar{\Omega}, \quad|x-\underline{x}|=\rho, \quad w(x) \geq M .
$$

Next take $x_{0} \in \overline{B(\underline{x}, \rho)} \cap \bar{\Omega}$ such that

$$
\left(v^{*}-w\right)\left(x_{0}\right)=\frac{\max }{B(\underline{x}, \rho) \cap \bar{\Omega}}\left(v^{*}-w\right) .
$$

There are three possible cases.

1. If $x_{0} \in \partial B(\underline{x}, \rho) \cap \bar{\Omega}$ then $w\left(x_{0}\right) \geq M \geq v^{*}\left(x_{0}\right)$. It follows that $v^{*}(x) \leq w(x)$ for $x \in \overline{B(\underline{x}, \rho)} \cap \bar{\Omega}$, which, taking $x$ in a neighborhood of $\underline{x}$, yields the contradiction $c \leq w(x) \leq m^{\prime}$.

2. If $x_{0} \in B(\underline{x}, \rho) \cap \Omega$ we use that $v^{*}$ is a subsolution of $F[u]=0$ to get $F[w]\left(x_{0}\right) \leq 0$, a contradiction with $F[w]>0$.

3. Finally, if $x_{0} \in B(\underline{x}, \rho) \cap \partial \Omega$, we use that $v^{*}$ is a viscosity subsolution of the boundary condition, namely, either $v^{*}\left(x_{0}\right) \leq \phi\left(x_{0}\right)$ or $F[w]\left(x_{0}\right) \leq 0$. The latter case is impossible because $F[w]>0$, whereas the former contradicts $\phi(x)<c \leq v^{*}(x)$ in $B(\underline{x}, \rho) \cap \partial \Omega$.

In all cases we reach a contradiction and complete the proof.

Remark 5.3. The conclusion of the last theorem still holds if the $C^{2}$ regularity of $\partial \Omega$ holds only in a neighborhood of $\underline{x}$, provided that (2.5) is replaced by (3.3) and (3.4). In fact the Liouville property still holds by Proposition 4.1.

\section{The ERgodic HJB EQUATION In INVARIANT BOUNDED DOMAins}

In this Section we will assume the stronger condition (2.8) on the behavior of the coefficients at the boundary of the domain $\Omega$, as well as the strict ellipticity of the operator $L$ in the interior of $\Omega(2.9)$. We will see in Section 6.2 that the assumption (2.8) is related to the invariance of both $\bar{\Omega}$ and $\Omega$ for the control system (1.5). 


\subsection{Well-posedness of the PDE}

This subsection is dedicated to the proof of Theorem 2.5. We set

$$
H(x, p, X):=\sup _{\alpha \in A}(-b(x, \alpha) \cdot p-\operatorname{tr}(a(x, \alpha) X)-l(x, \alpha)),
$$

where $l: \bar{\Omega} \times A \rightarrow \mathbb{R}$ is a bounded function satisfying (2.10). In order to find a solution to (1.3), we consider the approximated problems

$$
\left.\lambda u_{\lambda}(x)+H\left(x, D u_{\lambda}(x), D^{2} u_{\lambda}(x)\right)\right)=0, \quad x \in \Omega,
$$

for $\lambda>0$. We start with showing that this equation admits a unique viscosity solution, without prescribing any boundary conditions.

We recall the following well-known a priori estimates.

Lemma 6.1 (Krylov-Safonov estimates). Let assumptions (2.4), (2.9) and (2.10) hold and let $u \in C(\Omega)$ be a bounded viscosity solution to (6.2) with $\lambda>0$. Then $u \in C^{2}(\Omega)$, and for every compact set $K \subset \Omega$ there exists $\gamma \in(0,1)$, depending on the space dimension $n$, the ellipticity constants in $K$ and $\beta$ from (2.4) such that

$$
\|u\|_{C^{1, \gamma}(K)} \leq C_{K}
$$

with $C_{K}$ depending on $\gamma, K, \Omega,\|\sigma\|_{\infty}, B$ from $(2.4),\|b\|_{\infty},\|l\|_{\infty},\|u\|_{\infty}$ and any $\bar{\lambda} \geq \lambda$.

Proof. For the proof we refer to [32], Theorem 2.1 and section on further regularity, page 950 (see Sect. 17.4 in [24] and [31]).

Theorem 6.2. Under the assumptions (2.4), (2.8)-(2.10), for every $\lambda>0$, equation (6.2) admits a unique bounded solution $u_{\lambda} \in C^{2}(\Omega)$. Moreover for every $\tilde{x} \in \Omega$ and every $K \subset \subset \Omega$, the family $\left(u_{\lambda}-u_{\lambda}(\tilde{x})\right)_{\lambda \in(0,1]}$ is bounded in $C^{1, \gamma}(K)$, for some $\gamma \in(0,1)$. Finally, for all $h>0$, there exists $\delta_{h}>0$ such that

$$
\forall \lambda \in(0,1], x \in \Omega_{\delta_{h}}, \quad h \log (d(x))+\min _{\Omega \backslash \Omega_{\delta_{h}}} u_{\lambda} \leq u_{\lambda}(x) \leq-h \log (d(x))+\max _{\Omega \backslash \Omega_{\delta_{h}}} u_{\lambda} .
$$

Proof. The proof is divided into three parts.

Step 1. Existence. The idea is to apply Perron's method to the Neumann problem for (6.2), namely, under the boundary condition $\partial_{\nu} u=0$. Notice that the functions $\pm\|l\|_{\infty} / \lambda$ are sub and supersolutions for such problem. However, a technical difficulty in the comparison principle comes from the lack of Lipschitz-continuity of the terms $a, b$. Also, the application of Perron's method to this problem is achieved in [9] under the additional assumption that $\Omega$ is of class $W^{3, \infty}$. To overcome these difficulties one can proceed as follows. Consider a sequence of smooth approximations of the $a(\cdot, \alpha), b(\cdot, \alpha)$ and a sequence of smooth domains invading $\Omega$. The associated Neumann problems admit solution between $-\|l\|_{\infty} / \lambda$ and $\|l\|_{\infty} / \lambda$ by [9]. Finally, using the estimates provided by Lemma 6.1 and the stability of viscosity solutions, one can pass to the limit along a subsequence of such solutions and obtain a solution $u_{\lambda}$ to (6.2). Notice that the limit is only local in $\Omega$ and therefore we lose the information about the boundary behavior of $u_{\lambda}$. We only know that $u_{\lambda}$ is in $C^{2}(\Omega)$ and satisfies

$$
\forall x \in \Omega, \quad-\frac{\|l\|_{\infty}}{\lambda} \leq u_{\lambda}(x) \leq \frac{\|l\|_{\infty}}{\lambda} .
$$

Step 2. Uniqueness. Consider two bounded viscosity solutions $u, v \in C(\Omega)$ to (6.2). Then they are both in $C^{2}(\Omega)$ by Lemma 6.1 . We first modify $v$ in order to obtain a supersolution blowing up at the boundary. To this end, we will make use of the Lyapunov function $-\log (d(x))$ for the operator $F$ defined by (1.1). Take $\delta>0$ from Proposition 3.2, associated with $M=0$, and consider the function $-\log (d(x))$ defined for $x \in \Omega_{\delta}$. Let $V$ 
be a $C^{2}$ extension of this function to the whole $\Omega$. Up to replacing $\delta$ with $\min (\delta, 1)$, we have that $V \geq 0$ in $\Omega_{\delta}$. Then, for $\varepsilon>0$, define

$$
v_{\varepsilon}:=v+\varepsilon^{2} V+\varepsilon
$$

Using the fact that $v$ satisfies (6.2), we find that, for $x \in \Omega$,

$$
\begin{aligned}
\lambda v_{\varepsilon}(x)+H\left(x, D v_{\varepsilon}(x), D^{2} v_{\varepsilon}(x)\right) \geq & \lambda \varepsilon^{2} V(x)+\lambda \varepsilon \\
& +\varepsilon^{2} \inf _{\alpha \in A}\left(b(x, \alpha) \cdot D V(x)+\operatorname{tr}\left(a(x, \alpha) D^{2} V(x)\right)\right) \\
= & \varepsilon^{2}(\lambda V(x)-F[-V](x))+\varepsilon \lambda .
\end{aligned}
$$

This last expression is positive if $x \in \Omega_{\delta}$, because $F[-V]<0$ there. Otherwise, if $x \in \Omega \backslash \Omega_{\delta}$, it is larger than $\varepsilon(\lambda-C \varepsilon)$, where $C$ is a constant only depending on $n, \lambda$ and the $L^{\infty}$ norms of $a, b, V, D V, D^{2} V$ in $\Omega \backslash \Omega_{\delta}$. We deduce that $v_{\varepsilon}$ is a supersolution to (6.2) provided $\varepsilon$ is smaller than some $\varepsilon_{0}$. Now, since $\left(v_{\varepsilon}-u\right)(x) \rightarrow+\infty$ as $x \rightarrow \partial \Omega$, the function $v_{\varepsilon}-u$ attains its minimum on $\Omega$ at some point $y$. By regularity we have that $D v_{\varepsilon}(y)=D u(y)$ and $D^{2} v_{\varepsilon}(y) \geq D^{2} u(y)$. Therefore, if $\varepsilon \in\left(0, \varepsilon_{0}\right)$, we obtain

$$
\begin{aligned}
0 & \leq \lambda v_{\varepsilon}(y)+\sup _{\alpha \in A}\left(-b(y, \alpha) \cdot D v_{\varepsilon}(y)-\operatorname{tr}\left(a(y, \alpha) D^{2} v_{\varepsilon}(y)\right)-l(y, \alpha)\right) \\
& \leq \lambda\left(v_{\varepsilon}-u\right)(y)+\lambda u(y)+\sup _{\alpha \in A}\left(-b(y, \alpha) \cdot D u(y)-\operatorname{tr}\left(a(y, \alpha) D^{2} u(y)\right)-l(y, \alpha)\right) \\
& =\lambda\left(v_{\varepsilon}-u\right)(y) .
\end{aligned}
$$

It follows that $v_{\varepsilon} \geq u$ in $\Omega$ and thus $v \geq u$ in $\Omega$ by the arbitrariness of $\varepsilon \in\left(0, \varepsilon_{0}\right)$. Reversing the roles of $u, v$ we eventually derive $u \equiv v$.

Step 3. A priori bounds. We start with deriving (6.3). Fix $h>0$ and let $\delta_{h}$ be the $\delta$ given by Proposition 3.1 with $M=2\|l\|_{\infty} / h$. Set

$$
V(x):=-h \log (d(x))+\max _{\Omega \backslash \Omega_{\delta_{h}}} u_{\lambda} .
$$

It is not restrictive to assume that $\delta_{h} \leq 1$, so that $V(x) \geq u_{\lambda}(x)$ if $d(x)=\delta_{h}$. On the other hand, there exists $\delta^{\prime} \in\left(0, \delta_{h}\right)$ small enough, depending on $\lambda$, such that $V(x)>\|l\|_{\infty} / \lambda \geq u_{\lambda}(x)$ if $d(x)<\delta^{\prime}$. Finally, since $u_{\lambda} \geq-\|l\|_{\infty} / \lambda$ and $F[V]>h M=2\|l\|_{\infty}$, for $x \in \Omega_{\delta_{h}}$ we get

$$
\lambda V(x)+\sup _{\alpha \in A}\left(-b(x, \alpha) \cdot D V(x)-\operatorname{tr}\left(a(x, \alpha) D^{2} V(x)\right)-l(x, \alpha)\right) \geq \lambda \max _{\Omega \backslash \Omega_{\delta_{h}}} u_{\lambda}+F[V]-\|l\|_{\infty}>0 .
$$

It follows from the fact that $u_{\lambda}$ is a (sub) solution to (6.2) that

$$
\forall \delta \in\left(0, \delta^{\prime}\right), \quad \max _{\Omega_{\delta_{h}} \backslash \Omega_{\delta}}\left(u_{\lambda}-V\right)=\max _{\partial\left(\overline{\Omega_{\delta_{h}}} \backslash \Omega_{\delta}\right)}\left(u_{\lambda}-V\right) \leq 0 .
$$

Namely, $u_{\lambda} \leq V$ in $\Omega_{\delta_{h}}$, which is the second inequality in (6.3). The first inequality is obtained in analogous way, by using Proposition 3.2 in place of 3.1 and considering the subsolution $V(x):=\min _{\Omega \backslash \Omega_{\delta_{h}}} u_{\lambda}+h \log (d(x))$.

We now fix $\tilde{x} \in \Omega$ and we claim that the functions $\left(v_{\lambda}\right)_{\lambda \in(0,1]}$ defined by

$$
v_{\lambda}(x):=u_{\lambda}(x)-u_{\lambda}(\tilde{x})
$$

are equibounded in any $K \subset \subset \Omega$. Assume by way of contradiction that there exists $K \subset \subset \Omega$ such that

$$
\varepsilon_{\lambda}^{-1}:=\left\|v_{\lambda}\right\|_{L^{\infty}(K)} \rightarrow+\infty \quad \text { as } \lambda \rightarrow 0^{+} .
$$

Up to enlarging $K$ if needed, we can suppose that $\tilde{x} \in K$ and that, for $\delta_{1}$ from (6.3), $\Omega \backslash \Omega_{\delta_{1}} \subset K$. The function $\psi_{\lambda}(x):=\varepsilon_{\lambda} v_{\lambda}(x)$ satisfies $\left\|\psi_{\lambda}\right\|_{L^{\infty}(K)}=1, \psi_{\lambda}(\tilde{x})=0$ and

$$
\lambda \psi_{\lambda}+\lambda \varepsilon_{\lambda} u_{\lambda}(\tilde{x})+\sup _{\alpha \in A}\left(-b(x, \alpha) \cdot D \psi_{\lambda}(x)-\operatorname{tr}\left(a(x, \alpha) D^{2} \psi_{\lambda}(x)\right)-\varepsilon_{\lambda} l(x, \alpha)\right)=0, \quad x \in \Omega .
$$


Note that $\left|\lambda \varepsilon_{\lambda} u_{\lambda}(\tilde{x})\right| \leq \varepsilon_{\lambda}\|l\|_{\infty} \rightarrow 0$ as $\lambda \rightarrow 0$. Furthermore, by (6.3), for $x \in \Omega \backslash K$,

$$
\psi_{\lambda}(x)=\frac{u_{\lambda}(x)-u_{\lambda}(\tilde{x})}{\left\|u_{\lambda}-u_{\lambda}(\tilde{x})\right\|_{L^{\infty}(K)}} \leq \frac{\max _{\Omega \backslash \Omega_{\delta_{1}}} u_{\lambda}-\log (d(x))-u_{\lambda}(\tilde{x})}{\left\|u_{\lambda}-u_{\lambda}(\tilde{x})\right\|_{L^{\infty}(K)}} \leq 1-\varepsilon_{\lambda} \log (d(x)),
$$

and

$$
\psi_{\lambda}(x) \geq \frac{\min _{\Omega \backslash \Omega_{\delta_{1}}} u_{\lambda}+\log (d(x))-u_{\lambda}(\tilde{x})}{\left\|u_{\lambda}-u_{\lambda}(\tilde{x})\right\|_{L^{\infty}(K)}} \geq-1+\varepsilon_{\lambda} \log (d(x)) .
$$

A first consequence of these estimates is that, in any compact subset of $\Omega$, the $\psi_{\lambda}$ are equibounded, whence equibounded in $C^{1, \gamma}$ by Lemma 6.1. Using a diagonal procedure, we can then find a sequence $\lambda \rightarrow 0$ for which the $\psi_{\lambda}$ converge locally uniformly in $\Omega$ to some function $\psi \in C(\Omega)$. By stability property of viscosity solutions, $\psi$ solves

$$
\sup _{\alpha \in A}\left(-b(x, \alpha) \cdot D \psi(x)-\operatorname{tr}\left(a(x, \alpha) D^{2} \psi(x)\right)\right)=0, \quad x \in \Omega .
$$

We further know that $\|\psi\|_{L^{\infty}(K)}=1$, and, for $x \in \Omega \backslash K,|\psi(x)| \leq 1$ because $\left|\psi_{\lambda}(x)\right| \leq 1-\varepsilon_{\lambda} \log (d(x))$. This means that $\psi$ attains either the global maximum 1 or minimum -1 in $K$, and therefore it is constantly equal to 1 or -1 by the Strong Maximum or Minimum Principle, which holds by (2.9). This is impossible because $\psi(\tilde{x})=0$.

We have shown that the $v_{\lambda}$ are equibounded in any $K \subset \subset \Omega$. Since they satisfy (6.2) with $l$ replaced by $l+\lambda u_{\lambda}(\tilde{x})$, and $\left|\lambda u_{\lambda}(\tilde{x})\right| \leq\|l\|_{\infty}$, Lemma 6.1 eventually yields that they are equibounded in $C^{1, \gamma}(K)$.

We are now in the position to prove our main result.

Proof of Theorem 2.5. First of all we prove that there exists $c \in \mathbb{R}$ such that (1.3) admits a solution. Consider the solutions $\left(u_{\lambda}\right)_{\lambda \in(0,1]}$ to (6.2). Fix $\tilde{x} \in \Omega$. By (6.4), $\lambda u_{\lambda}(\tilde{x})$ converges (up to subsequences) to some value $-c$ as $\lambda \rightarrow 0$. Define $v_{\lambda}=u_{\lambda}-u_{\lambda}(\tilde{x})$. Theorem 6.2 gives that the $v_{\lambda}$ are equibounded in $C^{1, \gamma}(K)$, for any $K \subset \subset \Omega$. Thus, using a diagonalization procedure, we can extract a subsequence of $v_{\lambda}$ converging locally uniformly to $\chi \in C(\Omega)$, which, by stability, is a viscosity solution to (1.3). We know from Lemma 6.1 that $\chi \in C^{2}(\Omega)$. Moreover, $\chi$ satisfies the same bounds (6.3) as the $u_{\lambda}$ (and the $v_{\lambda}$ ). Using the fact that such bounds hold true for arbitrary $h>0$, we eventually find that $\chi$ fulfils (2.11). Uniqueness of $c$ and $\chi$ (up to constants) follows by the following stronger uniqueness result.

Proposition 6.3. Let $\chi_{1}$ and $\chi_{2}$ be viscosity solutions of (1.3) with, respectively, $c=c_{1}$ and $c=c_{2}$. Assume moreover there exist $\kappa>0$ such that

$$
\lim _{x \rightarrow \partial \Omega} \chi_{1}(x) d(x)^{\kappa}=0=\lim _{x \rightarrow \partial \Omega} \chi_{2}(x) d(x)^{\kappa} .
$$

Then $c_{1}=c_{2}$ and there exists a constant $k \in \mathbb{R}$ such that $\chi_{1} \equiv \chi_{2}+k$.

Proof. First of all, observe that by Lemma $6.1, \chi_{1}, \chi_{2} \in C^{2}(\Omega)$. Without loss of generality we can assume $c_{1} \geq c_{2}$. So,

$$
\sup _{\alpha \in A}\left(-b(x, \alpha) \cdot D\left(\chi_{1}-\chi_{2}\right)-\operatorname{tr}\left(a(x, \alpha) D^{2}\left(\chi_{1}-\chi_{2}\right)\right)\right) \geq c_{1}-c_{2} \geq 0 .
$$

By Theorem 4.2 and Remark 4.3, $\chi_{1}-\chi_{2}$ is a constant, and therefore $c_{1}=c_{2}$.

Remark 6.4. If we weaken the Hölder regularity (2.10) on the coefficients $b, l$ of equation (6.2) to the uniform continuity as stated in (2.3), Krylov Safonov estimates stated in Lemma 6.1 still hold, but we just expect that any bounded continuous viscosity solution to $(6.2)$ is $C^{1, \alpha}(\Omega)$ for every $\alpha \in(0,1)$, not in $C^{2}(\Omega)$.

This implies that in Theorem 6.2 and in Theorem 2.5 the solutions $u_{\lambda}$ to (6.2) and $\chi$ to (1.3) are of class $C^{1, \alpha}(\Omega)$ for every $\alpha \in(0,1)$. So, in the step Uniqueness of the proof of Theorem 6.2 the argument has to be modified by using the by now standard doubling variables argument in the theory of viscosity solutions (see e.g. [21]). Moreover, also in the proof of Theorem 2.5, the argument to prove that $\chi_{1}-\chi_{2}$ solves (6.5) has to be modified appropriately. 


\subsection{A stochastic control interpretation}

We show an application of the previous results to an ergodic control problem in $\Omega$ with state constraints. Throughout this subsection, we will assume the stronger regularity assumptions on the coefficients (5.1) to hold. It is known from $[5,7]$ that, under the assumption $(2.8)$ on the behavior of the coefficients near $\partial \Omega$, the set $\bar{\Omega}$ is invariant for the control system (1.5) in the following sense: for every $x \in \bar{\Omega}$ and any admissible control $\alpha$. $\in \mathcal{A}$, the trajectory of (1.5) satisfies $X_{t}^{\alpha \cdot} \in \bar{\Omega}$ almost surely for all $t \geq 0$.

Therefore no restrictions on the controls are needed to keep the system forever in $\bar{\Omega}$, and we can define the value function of the infinite horizon discounted control problem with state constraint $\bar{\Omega}$

$$
v_{\lambda}(x):=\inf _{\alpha . \in \mathcal{A}} \mathbb{E}\left[\int_{0}^{\infty} \mathrm{e}^{-\lambda t} l\left(X_{t}^{\alpha .}, \alpha_{t}\right) \mathrm{d} t\right], \quad x \in \bar{\Omega}
$$

where $l: \bar{\Omega} \times A \rightarrow \mathbb{R}$ is a bounded function satisfying (2.3) and $\lambda>0$. The next result states that $v_{\lambda}$ is the solution of the PDE (6.2).

Proposition 6.5. Let (2.8) and (5.1) hold. Then $v_{\lambda}$ is continuous in $\bar{\Omega}$ and it is a viscosity solution of (6.2).

If, in addition, (2.9) holds, then $v_{\lambda}(x)=u_{\lambda}(x)$ for all $x \in \Omega$, where $u_{\lambda}$ is the smooth solution of (6.2) given by Theorem 6.2 .

Proof. Fix $\epsilon>0$ and $T$ large enough so that $\int_{T}^{\infty} \mathrm{e}^{-\lambda t} l\left(X_{t}^{\alpha .}, \alpha_{t}\right) \mathrm{d} t<\epsilon$ for all $x$ and $\alpha$.. Pick $x, y \in \bar{\Omega}$ and a control $\alpha$. $\in \mathcal{A} \epsilon$-optimal for the initial point $y$. Then, denoting with $Y_{t}^{\alpha}$. the trajectory starting form $y$ and using such control,

$$
v_{\lambda}(x)-v_{\lambda}(y) \leq \mathbb{E}\left[\int_{0}^{T} \mathrm{e}^{-\lambda t}\left|l\left(X_{t}^{\alpha \cdot}, \alpha_{t}\right)-l\left(Y_{t}^{\alpha .}, \alpha_{t}\right)\right| \mathrm{d} t\right]+3 \epsilon .
$$

Now we use the standard estimate $\mathbb{E}\left[\left|X_{t}^{\alpha \cdot}-Y_{t}^{\alpha \cdot}\right|\right] \leq \mathrm{e}^{B t}|x-y|$ and the assumption $|l(X, \alpha)-l(Y, \alpha)| \leq \omega|X-Y|$, where the modulus $\omega$ can be assumed to be concave w.l.o.g., to get

$$
v_{\lambda}(x)-v_{\lambda}(y) \leq \int_{0}^{T} \mathrm{e}^{-\lambda t} \omega\left(\mathrm{e}^{B t}|x-y|\right) \mathrm{d} t+3 \epsilon
$$

and the right hand side can be made smaller than $4 \epsilon$ by choosing $|x-y|$ small enough. Then the continuity of $v_{\lambda}$ is obtained by repeating the argument with the roles of $x$ and $y$ reversed. Once this is established the Dynamic Programming Principle is a standard result, as well as deducing from it that $v_{\lambda}$ solves the equation in $\Omega$ in viscosity sense, see, e.g., [23].

The last statement follows from Theorem 6.2.

Next we show that under the conditions (5.1) and (2.8) of this section also the open set $\Omega$ is invariant for the control system (1.5), in analogy with Proposition 5.1.

Proposition 6.6. Assume (2.8) and (5.1). Then, for every $x \in \Omega$ and every admissible control $\alpha ., X_{t}^{\alpha .} \in \Omega$ almost surely for all $t \geq 0$, i.e. $\tau_{x}^{\alpha .}=+\infty$ almost surely, where $\tau_{x}^{\alpha .}$ is defined in (5.3).

Proof. The proof follows the same arguments as in the proof of Proposition 5.1. Let $U$ be a $C^{2}$ extension of the function $\log (d(x))$ to the whole $\Omega$, which coincides with $\log d(x)$ in a neighborhood of $\partial \Omega$. We can assume that $U \leq-1$ in $\Omega$ and moreover, by Proposition 3.6, there exists a constant $C \geq 0$ such that

$$
F[U](x) \leq C \quad x \in \Omega .
$$

By suboptimality principles for viscosity solutions (see [19]), we get for every $\delta>0$, every $x \in \Omega \backslash \Omega_{\delta}$ and every $t \geq 0$, recalling that $V \leq-1$,

$$
U(x) \leq \inf _{\alpha \cdot \in \mathcal{A}} \mathbb{E}\left[U\left(X_{\tau_{x}^{\delta, \alpha \cdot \wedge t}}^{\alpha .}\right)+C\left(\tau_{x}^{\delta, \alpha \cdot \wedge t}\right)\right]
$$


and then for all $x \in \Omega$, and all $\delta>0$

$$
\sup _{\alpha . \in \mathcal{A}} \mathbb{P}\left(\omega \mid \tau_{x}^{\delta, \alpha \cdot}(\omega) \leq t\right) \leq \frac{C t-U(x)}{-\log \delta} .
$$

So, for every $t \geq 0$,

$$
\sup _{\alpha . \in \mathcal{A}} \mathbb{P}\left(\omega \mid \tau_{x}^{\alpha \cdot}(\omega) \leq t\right)=0 .
$$

From this we conclude as in the proof of Proposition 5.1 that $\mathbb{P}\left(\omega \mid \tau_{x}^{\alpha .}(\omega)<+\infty\right)=0$ for every $\alpha$. $\in \mathcal{A}$.

This result allows us to interpret $u_{\lambda}(x)=v_{\lambda}(x)$ with $x \in \Omega$ also as the value of the discounted infinite horizon problem with state constraint the open set $\Omega$. We can also give a representation of $u_{\lambda}$ that is more consistent with the method of construction by means of Neumann boundary conditions used in Theorem 6.2. In fact, the condition $\partial_{\nu} u=0$ on $\partial \Omega$ is related with the optimal control of systems for the state equation

$$
\left\{\begin{array}{l}
\mathrm{d} X_{t}^{\alpha .}=b\left(X_{t}^{\alpha .}, \alpha_{t}\right) \mathrm{d} t+\sqrt{2} \sigma\left(X_{t}^{\alpha .}, \alpha_{t}\right) \mathrm{d} W_{t}-\nu\left(X_{t}^{\alpha .}\right) \mathrm{d} k_{t}, \quad X_{0}^{\alpha .}=x \in \bar{\Omega} \\
k_{t}=\int_{0}^{t} \mathbb{1}_{\partial \Omega}\left(X_{s}^{\alpha .}\right) \mathrm{d} k_{s} \quad \text { is nondecreasing, }
\end{array}\right.
$$

where $X_{t}^{\alpha}$ and $k_{t}$ are adapted continuous processes, $\nu$ is the unit outward normal to $\partial \Omega$, and $\mathbb{1}_{\partial \Omega}$ is the indicator function of $\partial \Omega$. This is a controlled diffusion process with normal reflection at the boundary (see, e.g., [3]). By Proposition 6.6, if $x \in \Omega$, the trajectory of (6.7) corresponding to a given control $\alpha$. $\in \mathcal{A}$ coincides a.s. with the trajectory of (1.5) associated with the same control. Therefore the solution $u_{\lambda}$ of the PDE (6.2) is also the value function of the discounted infinite horizon problem for the system (6.7) with trajectories reflected at the boundary.

In conclusion we obtain a stochastic representation formula for the solution pair $c, \chi$ of the ergodic Bellman equation (1.3).

Corollary 6.7. Let (2.8), (2.9), (5.1), hold and let $v_{\lambda}$ be defined by (6.6) with $X_{t}^{\alpha .}$ solving either (1.5) or (6.7). Then the constant $c$ of Theorem 2.5 satisfies

$$
c=\lim _{\lambda \rightarrow 0+} \lambda v_{\lambda}(x) \quad \forall x \in \Omega,
$$

and for any $\tilde{x} \in \Omega$, a solution of (1.3) corresponding to $c$ is

$$
\chi(x)=\lim _{\lambda \rightarrow 0+}\left(v_{\lambda}(x)-v_{\lambda}(\tilde{x})\right), \quad x \in \Omega,
$$

where the convergence is locally uniform in $\Omega$ in both limits.

The formula (6.8) shows a connection with ergodic control, since the limits

$$
\lim _{\lambda \rightarrow 0+} \lambda \mathbb{E}\left[\int_{0}^{\infty} \mathrm{e}^{-\lambda t} l\left(X_{t}^{\alpha .}, \alpha_{t}\right) \mathrm{d} t\right], \quad \lim _{T \rightarrow+\infty} \frac{1}{T} \mathbb{E}\left[\int_{0}^{T} l\left(X_{t}^{\alpha .}, \alpha_{t}\right) \mathrm{d} t\right]
$$

coincide if either one exists, by classical Abelian-Tauberian theorems.

\section{REFERENCES}

[1] O. Alvarez and M. Bardi, Ergodicity, stabilization, and singular perturbations for Bellman-Isaacs equations. Mem. Amer. Math. Soc. 204 (2010) 960.

[2] A. Arapostathis, V.S. Borkar and M.K. Ghosh, Ergodic control of diffusion processes. Cambridge University Press, Cambridge (2012).

[3] M. Arisawa and P.-L. Lions, On ergodic stochastic control. Commun. Partial Differ. Equ. 23 (1998) $2187-2217$. 
[4] M. Bardi and I. Capuzzo-Dolcetta, Optimal control and viscosity solutions of Hamilton-Jacobi-Bellman equations. Birkhäuser Boston, Boston, MA (1997).

[5] M. Bardi and P. Goatin, Invariant sets for controlled degenerate diffusions: a viscosity solutions approach, in "Stochastic Analysis, Control, Optimization and Applications: A Volume in Honor of W.H. Fleming", edited by W.M. McEneaney, G.G. Yin and Q. Zhang. Birkhäuser, Boston (1999) 191-208.

[6] M. Bardi and F. Da Lio, Propagation of maxima and strong maximum principle for fully nonlinear degenerate elliptic equations. Part I: convex operators. Nonlinear Anal. 44 (2001) 991-1006.

[7] M. Bardi and R. Jensen, A geometric characterization of viable sets for controlled degenerate diffusions. Set-Valued Anal. 10 (2002) 129-141.

[8] M. Bardi and F. Da Lio, Propagation of maxima and strong maximum principle for fully nonlinear degenerate elliptic equations. Part II: concave operators. Indiana Univ. Math. J. 52 (2003) 607-627.

[9] G. Barles, Fully nonlinear Neumann type boundary conditions for second-order elliptic and parabolic equations. J. Differ. Equ. 106 (1993) 90-106.

[10] G. Barles and J. Burdeau, The Dirichlet problem for semilinear second-order degenerate elliptic equations and applications to stochastic exit time control problems. Commun. Partial Differ. Eq. 20 (1995) 129-178.

[11] G. Barles and E. Rouy, A strong comparison result for the Bellman equation arising in stochastic exit time control problems and its applications. Commun. Partial Differ. Equ. 23 (1998) 11-12, 1995-2033.

[12] G. Barles and F. Da Lio, On the boundary ergodic problem for fully nonlinear equations in bounded domains with general nonlinear Neumann boundary conditions. Ann. Inst. Henri Poincaré Anal. Non Linéaire 22 (2005) 521-541.

[13] G. Barles, A. Porretta and T.T. Tchamba, On the large time behavior of solutions of the Dirichlet problem for subquadratic viscous Hamilton-Jacobi equations. J. Math. Pures Appl. 94 (2010) 497-519.

[14] A. Bensoussan and J. Frehse, Ergodic control Bellman equation with Neumann boundary conditions. Vol. 280 of Lect. Notes Control Inform. Sci. Springer, Berlin (2002) 59-71.

[15] H. Berestycki, I. Capuzzo Dolcetta, A. Porretta, L. Rossi, Maximum Principle and generalized principal eigenvalue for degenerate elliptic operators. J. Math. Pures Appl. 103 (2015) 1276-1293.

[16] V. Borkar and A. Budhiraja, Ergodic control for constrained diffusions: characterization using HJB equations. SIAM J. Control Optim. 43 (2004/05) 1467-1492.

[17] P. Cannarsa, G. Da Prato and H. Frankowska, Invariant measures associated to degenerate elliptic operators. Indiana Univ. Math. J. 59 (2010) 53-78.

[18] I. Capuzzo Dolcetta, A. Cutrì, Hadamard and Liouville type results for fully nonlinear partial differential inequalities. Commun. Contemp. Math. 5 (2003) 435-448.

[19] A. Cesaroni, Lyapunov Stabilizability of Controlled Diffusions via a Superoptimality Principle for Viscosity Solutions. Appl. Math. Optim. 53 (2006) 1-29.

[20] H. Chen and P. Felmer, On Liouville type theorems for fully nonlinear elliptic equations with gradient term. J. Differ. Eq. 255 (2013) 2167-2195.

[21] M.G. Crandall, H. Ishii and P.-L. Lions, User's guide to viscosity solutions of second order partial differential equations. Bull. Amer. Math. Soc. (N.S.) $\mathbf{2 7}$ (1992) 1-67.

[22] G. Fichera, Sulle equazioni differenziali lineari ellittico-paraboliche del secondo ordine. Atti Accad. Naz. Lincei. Mem. Cl. Sci. Fis. Mat. Nat. Sez. I. 5 (1956) 1-30.

[23] W.H. Fleming and H.M. Soner, Controlled Markov processes and viscosity solutions. 2nd edition. Springer, New York (2006).

[24] D. Gilbarg, N.S. Trudinger, Elliptic Partial Differential Equations of Second Order. Springer, Berlin (1988).

[25] U.G. Haussmann and J.-P. Lepeltier, On the existence of optimal controls. SIAM J. Control Optim. 28 (1990) 851-902.

[26] J.M. Lasry and P.-L. Lions, Nonlinear elliptic equations with singular boundary conditions and stochastic control with state constraints. I. The model problem. Math. Ann. 283 (1989) 583-630.

[27] T. Leonori and A. Porretta, Gradient bounds for elliptic problems singular at the boundary. Arch. Ration. Mech. Anal. 202 (2011) 663-705.

[28] P.-L. Lions, Quelques remarques sur les problèmes elliptiques quasilinéaires du second ordre. J. Anal. Math. 45 (1985) $234-254$.

[29] P.-L. Lions and C. Villani, Régularité optimale de racines carrées. C. R. Acad. Sci. Paris Sér. I Math. 321 (1995) $1537-1541$.

[30] A. Porretta, The "ergodic limit" for a viscous Hamilton-Jacobi equation with Dirichlet conditions. Atti Accad. Naz. Lincei Cl. Sci. Fis. Mat. Natur. Rend. Lincei (9) Mat. Appl. 21 (2010) 59-78.

[31] M.V. Safonov, On the classical solution of Bellman's elliptic equation. Sov. Math. Dokl. 30 (1984) $482-485$.

[32] N.S. Trudinger, On regularity and existence of viscosity solutions of nonlinear second order, elliptic equations. Partial differential equations and the calculus of variations. Vol. II of Progr. Nonlin. Differ. Equ. Appl. Birkhäuser Boston, Boston, MA (1989) 939-957. 